binding capacity, is also relatively modest. Gel electrophoresis in SDS gives a wide choice of protein zones, but a peak of 28,000 molecular weight is prominent and others are fairly clearly in evidence at 45,000 and 38,000 . Schmidt and Raftery proclaim their confidence, nevertheless, that the first of these is the genuine article, and in spite of the small subunit weight that this implies, take their courage in both hands and commit themselves to the assertion that previous estimates, for both Torpedo and electric eel receptor, of $40-50,000$ are incorrect.

It might perhaps be remarked that, although the isolation of the pure receptor is clearly altogether a good aim, many of its properties, particularly, as Franklin and Potter (FEBS Lett., 28, 101 ; 1972) have shown, its interaction with such ligands as bungarotoxin, can be usefully studied on the homogenized membrane particles.

Regarding the question of molecular weight and subunit structure, it may be noted that a different membrane-bound receptor - that for adrenergic molecules in heart-has been purified by affinity chromatography on columns containing a bound norepinephrine derivative (Lefkowitz, Haber and O'Hara, Proc. US Nat. Acad. Sci., 69, 2828 ; 1972), and comes in two sizes, peaks corresponding to about 40,000 and 160,000 molecular weight being eluted. The simplest explanation is that different states of aggregation of the receptor protein coexist in the membrane.

\section{PALYNOLOGY Making Pollen Diagrams}

from our Plant Ecology Correspondent AN article by A. D. Gordon and H. J. B. Birks in the current issue of New Phytologist (71, 961; 1972) provides new hope for the introduction of objective techniques into the interpretation of data obtained by pollen analysis of Quaternary deposits.

There are many statistical problems inherent in the presentation and interpretation of pollen data and only one of these is tackled here, namely the construction of an objectively defined zonation system for a pollen diagram. Before this can be done one must assume that the original data are an adequate representation of the pollen content of the sediment undergoing analysis and that the data are basically reliable. One must also assume that the sedimentary sequence of a deposit reflects its temporal development; in other words, that the stratified sediments have not been disturbed subsequent to their deposition.

For several decades it has been the practice of palynologists to divide their data into subsidiary groups, or zones, to facilitate the task of describing temporal changes in pollen proportions and the vegetational developments which these changes reflect. The use of zonation systems has been long established (for example, Godwin, New Phytol., 39, 370; 1940), but only recently have there been attempts to define the criteria which should be used for the subdivision of pollen diagrams (for example, Birks, J. Ecol., 58, 827 ; 1970).

The problem of objective zonation has been approached by using computerized classificatory techniques similar to those applied to vegetational analysis (for example, Dale and Walker, Pollen Spores, 12, 21 ; 1970), but these have usually been of limited value because of the absence of any stratigraphical constraint written into the programme. This has allowed the possibility of grouping samples together from different parts of the profile on the basis of their similar pollen spectra. Gordon and Birks have overcome this problem by requiring that samples grouped together in a zone should be stratigraphically contiguous.

They use a variety of techniques, both divisive and agglomerative, to construct objective zonation schemes for published data from Britain and the United States. The zones resulting from these analyses are in remarkable agreement with the subjective decisions of the original authors regardless of the precise numerical technique used. This study, in addition to the ratification which it provides for the intuitive divisions produced by earlier workers, also offers a means of objective zonation for future work.

There are some major disadvantages in this type of approach; first, the selection by the computer of abrupt rather than gradual changes as the basis for zone boundaries; second, the tendency to select zones defined by local rather than regional pollen types; and, third, the inability of the computer to place additional emphasis upon changes in species of critical ecological importance (for example, elm and plantain). In spite of these residual problems, Gordon and Birks's study represents an important step towards the removal of palynology from the realms of mysticism.

\section{NUCLEAR PHYSICS Ambiguity Resolved}

from a Correspondent ANOTHER way of removing the so-called "discrete ambiguities" of nuclear physics has been discovered. It involves the scattering of $\alpha$ particles from aligned nuclei.

Elastic $\alpha$ particle scattering can be very well described using the optical model of the interaction. According to this model, the $\alpha$ particle moves in a complex potential with radius and surface diffuseness similar to that of the nucleus itself, and with appropriate choice of parameters the differential cross-section can be fitted to high accuracy. Unfortunately, it is frequently found that there are several different sets of potentials which give equally good fits to the data ("discrete" ambiguities), and within each set it is often possible to make correlated variations of the parameters that preserve the fit ("continuous" ambiguities). Physically this is easily understood; elastic scattering depends only on the asymptotic form of the wavefunction and in addition the $\alpha$ particles interact so strongly with the nuclear field that those that are elastically scattered can only have passed through the surface region, so that all potentials giving the correct behaviour in this surface region give the same scattering.

\title{
Separating Myosin Functions
}

Iv next Wednesday's Nature New Biology (December 13), Silverman, Eisenberg and Kielley describe a study of the consequences of blocking one thiol group $\left(\mathrm{SH}_{1}\right)$ in the heavy chains of myosin. It was previously reported that these groups could be reacted with $\mathrm{N}$-ethylmaleimide, and the resulting protein was inhibited in regard to EDTA-ATPase activity, whereas the calcium-ATPase was enhanced.

Working with the more tractable molecule, heavy meromyosin (HMM), Silverman et al. have found that $\mathrm{SH}_{1-}$ blocked protein is activated by actin, but by a factor of only three, compared with 200 for unmodified HMM. From the dependence of this small activation on the actin concentration, the authors exclude any likelihood that the activation effect arises from a contaminant of unmodified HMM.

Silverman et al. have also investigated the effect of the relaxing system, troponin and tropomyosin, on the activity of the modified acto-HMM complex. In the absence of calcium the activation of ATPase by actin is completely suppressed, just as in normal actomyosin. It is concluded that the $\mathrm{SH}_{1}$ thiol group is required not only for EDTA-ATPase activity, but also for activation of magnesium ATPase by actin, whereas it is not involved in the interaction with the troponintropomyosin system. 
These ambiguities lead to difficulties when the potentials are used to generate wavefunctions for use in reaction calculations, so many attempts have been made to resolve them. In the case of deuterons, the low binding energy makes it easy to calculate the deuteronnucleus potential from the constituent neutron-nucleus and proton-nucleus optical potentials, but for the more tightly bound helions, tritons and $\alpha$ particles this cannot yet be done with sufficient accuracy. The discrete ambiguities tend to disappear at higher energies, and this can be understood physically (see Nature Physical Science, 239, 66; 1972).

Parks et al. (Phys. Rev. Lett., 29, 1264 ; 1972) have found another means of getting round the problem. Scattering from aligned nuclei is much more sensitive to the poteritial than the scattering from non-aligned nuclei-providing, of course, that the nucleus itself, and hence the potential, is deformed -and in their experiment, nuclei of the strongly-deformed ${ }^{165} \mathrm{Ho}$ were aligned by reducing the temperature to within a fraction of a degree of absolute zero. At these temperatures the forces within the metallic crystal align the nuclei, and this alignment is not destroyed by thermal motion.

The measurements were made by comparing the cross-section at $0.26 \mathrm{~K}$, when the nuclei were aligned, with that at $1.75 \mathrm{~K}$ when they were not. Then the quantity

$\frac{\Delta \sigma}{\sigma} \equiv \frac{\sigma(0.26 \mathrm{~K})-\sigma(1.75 \mathrm{~K})}{\sigma(1.75 \mathrm{~K})}=\sum_{m>0} A_{m} \sigma_{m}(\theta)$

gives a measure of the effects of the alignment on the scattering. This is shown in the diagram for incident $\alpha$ particle energies from 20 to $24 \mathrm{MeV}$ for scattering through $160^{\circ}$. It is also expressible as a sum over the cross-sections $\sigma_{m}(\theta)$ for scattering by the nucleus in a magnetic substate $m$. The coefficients $A_{m}$ can be calculated from the temperature of the crystal, its structure and the parameters of the nuclear hyperfine Hamiltonian, and $\sigma_{m}(\theta)$ can be obtained from a coupledchannels calculation of the interaction.

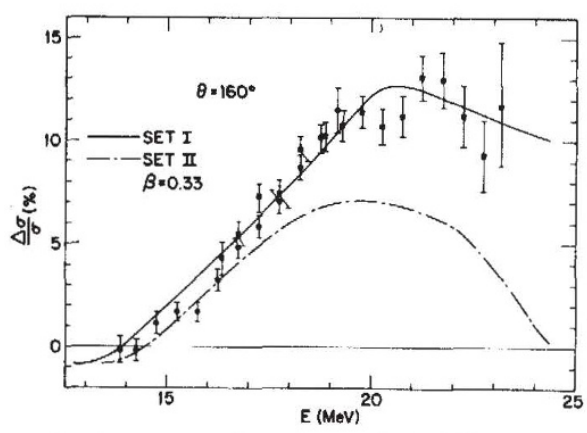

$\Delta \sigma / \sigma$ as a function of incident $\alpha$ particle energy. The two curves are calculated from optical potentials that give equally good fits to the differential cross-sections.
Thus $\Delta \sigma / \sigma$ can be calculated from the optical potential, and results of two such calculations, using potentials found by Aponick et al. (Nucl. Phys., A159, $367 ; 1970)$ to give equally good fits to the differential cross-section, are also shown in the figure.

It is clear that one is consistent with the data and the other is not, so that the discrete ambiguity is resolved. The best value of the nuclear deformation found by optimizing the fit to the data $(\beta=0.320 \pm 0.020)$ agrees well with the results found by analyses of Coulomb excitation $(\beta=0.33)$ and photoneutron cross-sections $(\beta=0.319 \pm 0.003)$. The sign of $\beta$ is determined because $\Delta \sigma / \sigma$ would have opposite sign for an oblate deformation.

\section{ELEMENTARY PARTICLES Muon Ranges Differ}

MEASUREMENTS on positive and negative muons made at the Lawrence Berkeley Laboratory of the University of California show that negative muons with a momentum of a few hundred $\mathrm{MeV}$ can travel about 0.2 per cent further in steel than can their positive counterparts of the same momentum. The experiment is reported by Clark $e t$ al. (Phys. Lett., 41B, 229 ; 1972) and can be explained in terms of a small correction to the familiar Bethe-Bloch formula for the rate of loss of energy of a singly charged particle.

Although measurements of range have long been used to estimate momenta, or, given separate measurements of momenta (by means of the magnetic deflexion method, for example), to distinguish particles of differing mass, it is only recently that accuracies of the order of fractions of a per cent in range measurements have become critical to the outcome of some CP violation experiments. And in the past cosmic ray physicists have usually been content with much larger accuracies.

Clark et al. used an experimental arrangement set up to look for the rare decay mode $\mathrm{K}_{\mathrm{L}}^{\mathrm{o}} \rightarrow \mu^{+}+\mu^{\sim}$. With this

\section{Two New Linkage Groups in Man}

Genetic analysis of somatic cell hybrids provides an increasing return as more data accumulate. In next Wednesday's Nature New Biology (December 13) van Someren, Mera Khan, Westerveld and Bootsma describe two new linkage groups in man. The information presented extends their previously published analysis of hybrids derived from fusions between Chinese hamster and human cells.

The novel contribution of the report concerns the relationship of the loci controlling, or coding for, glutamate pyruvate transaminase (GPT) and lactate dehydrogenase (LDH). GPT in red cells is polymorphic; two common and at least four rare alleles determined by an autosomal locus have been reported by Shi-Han Chen et al. Van Someren et al., however, conclude from their observations on this enzyme in human fibroblasts and leucocytes that two additional loci exist which determine interacting subunits referred to as GPT-B and GPT-C (to distinguish them from the red cell form GPT-A). Electrophoretic analysis of white cell extracts revealed five bands of GPT activity clearly different from the red cell enzyme. The five-banded appearance is suggestive of a tetrameric structure and this, together with the genetic evidence implicating two loci, indicates a close parallel to the lactate dehydrogenase system. The subunits of the latter enzyme, LDH-A and LDH-B, can either interact to give a tetramer comprising combinations of two subunit types or form exclusive associates of only one type. The GPT forms determined by the loci $g p t B$ and $g p t C$ can apparently associate in an analogous fashion.

Analysis of the electrophoretic patterns for LDH and GPT in extracts of the hybrid clones indicates that the loci for GPT-C and LDH-A and those for GPT-B and LDH-B are most probably linked. (About one-third of the sixtyeight clones analysed retained detectable GPT-B and LDH-B, the remainder having lost both. A similar proportion were positive for GPT-C and LDH-A. No disruption was observed for either linkage group.) Ruddle ( $A d v$. Hum. Genet., 4, 173; 1972) has assigned the loci for LDH-A and LDH-B to chromosomes 11 and 12 respectively. Thus the data support the localization of the different LDH and GPT loci on two chromosomes which are morphologically similar.

Van Someren et al. suggest that these observations support the theory that gene duplication (in this instance resulting from chromosome duplication) may have given rise to enzyme subunits which although electrophoretically distinguishable are functionally similar and able to cooperate. Further analysis of markers on these and other chromosomes is obviously required if this suggestion can be considered more than speculation. Detailed genetic analysis of the type reported, however, represents one of the most direct ways to evaluate the possible involvement of chromosome duplication in the evolution of the human karyotype. 